\title{
Dynamics and flavor symmetry realizations in chiral QCD
}

\author{
Kenichi Konishi* ${ }^{* \dagger}$ \\ Department of Physics, University of Pisa, Largo Pontecorvo, 3, 56127, Pisa, Italy, \\ INFN, Pisa Section, Largo Pontecorvo, 3 , 56127 Pisa, Italy \\ E-mail: kenichi.konishieunipi.it
}

The phases and symmetries of a wide class of strongly-interacting chiral gauge theories are investigated. The basic constraints arise from the 't Hooft anomaly matching condition, which is often difficult to satisfy, and as a consequence, restricts severely possible infrared behavior of the systems. Two mechanisms, the color-flavor locking and dynamical Abelianization, acting singly or in combination, emerge from our analysis as powerful ideas which help to find a solution.

XIII Quark Confinement and the Hadron Spectrum - Confinement2018

31 July - 6 August 2018

Maynooth University, Ireland

\footnotetext{
*Speaker.

${ }^{\dagger}$ Talk based on [1] and on another work in preparation with S. Bolognesi.
} 


\section{Introduction}

Our world has a nontrivial chiral structure. The macroscopic structures such as biological bodies often have approximately left-right symmetric forms, but not exactly. At the molecular level, $O\left(10^{-6} \mathrm{~cm}\right)$, the structure of DNA has a definite chiral spiral form. At the microscopic length scales of the fundamental interactions, $O\left(10^{-16} \mathrm{~cm}\right)$, the left- and right-handed quarks and leptons have different couplings to the $S U(3) \times S U_{L}(2) \times U_{Y}(1)$ gauge bosons.

In spite of these, and in spite of an almost half-century of studies of vectorlike gauge theories like $S U$ (3) quantum chromodynamics (QCD), based on straightforward approximate calculations (lattice simulations), and despite beautiful theoretical developments in models with $\mathscr{N}=2$ supersymmetries (which are all vectorlike), surprisingly little is known today about strongly-coupled chiral gauge theories. ${ }^{1}$ Perhaps it is not senseless to try to understand better this class of gauge theories, which Nature might be making use of, in a way as yet unknown to us.

To be concrete, we shall limit ourselves to $S U(N)$ gauge theories with a set of Weyl fermions in a complex representation of $S U(N)$. Also only asymptotically free models will be considered, as weakly coupled infrared-free theories can be reliably analyzed in perturbation theory, as in the case of the standard electroweak model.

The gauge interactions in these models become strongly coupled in the infrared. There are no gauge-invariant bifermion condensates, no mass terms or potential terms (of renormalizable type) can be added to deform the theories, no $\theta$ parameter exists. No center symmetry is present. The vacuum is unique.

For simplicity we shall restrict ourselves to various irreducible ${ }^{2} S U(N)$ chiral theories, with $N_{\psi}$ fermions $\psi^{\{i j\}}$ in the symmetric representation, $N_{\chi}$ fermions $\chi_{[i j]}$ in the anti anti-symmetric representation, and a number of antifundamental (or fundamental) multiplets, $\eta_{i}^{a}$ (or $\tilde{\eta}^{a i}$ ). The number of the latter is fixed so that the gauge group be anomaly free. We call these $\left(N_{\psi}, N_{\chi}\right)$ model. The question of our interest are:

(i) Does the system confine?

(ii) Does the system experience a dynamical Higgs phenomenon?

(iii) Does the system flow into an IR fixed-point CFT?

(iv) Does the chiral flavor symmetry remain unbroken, or if spontaneously broken, how?

(v) If there are more than one apparently possible dynamical scenarios, all consistent with e.g., 't Hooft anomaly matching conditions [2], the $a$ "theorem" [3], or other criteria such as the ACS condition [4], which one is actually realized in the infrared?

(vi) Does the system dynamically generate hierarchically disparate mass scales, such as in the "tumbling" scenarios [5]?

(vii) Do the systems simplify in the large $N$ limit, or is a planar equivalence to $\mathscr{N}=1$ supersymmetric models valid (e.g., [9])?

\footnotetext{
${ }^{1}$ For a partial list of references on the earlier studies of chiral theories, see [5]-[8].

${ }^{2}$ Namely we do not consider addition of fundamental-antifundamental pairs of fermions. The models of this type, in the simplest cases $\left(N_{\psi}, N_{\chi}\right)=(1,0),(0,1)$, have been studied in [4].
} 


\section{2. $\left(N_{\psi}, N_{\chi}\right)=(1,1)(" \psi \chi \eta ")$ model}

First consider the $(1,1)$ model, with left-handed fermion matter fields

$$
\psi^{\{i j\}}, \quad \chi_{[i j]}, \quad \eta_{i}^{A}, \quad A=1,2, \ldots 8,
$$

a symmetric tensor, an (anti-)antisymmetric tensor and eight anti-fundamental multiplets of $S U(N)$. The unbroken global symmetry is

$$
G_{F}=S U(8) \times U_{1}(1) \times U_{2}(1) \times Z_{N^{*}}
$$

$U_{1,2}(1)$ are anomaly free combinations of $U_{\psi}(1), U_{\chi}(1), U_{\eta}(1)$, and $Z_{N^{*}}$ is some discrete symmetry. They can be taken e.g., as

$$
\begin{array}{lll}
U_{1}(1): & \psi \rightarrow e^{i \frac{\alpha}{N+2}} \psi ; & \eta \rightarrow e^{-i \frac{\alpha}{8}} \eta ; \\
U_{2}(1): & \psi \rightarrow e^{i \frac{\beta}{N+2}} \psi ; & \chi \rightarrow e^{-i \frac{\beta}{N-2}} \chi .
\end{array}
$$

Various possible dynamical possibilities have been discussed in [1].

(i) Confinement phase with no chiral symmetry breaking is excluded by impossibility of finding massless baryons, saturating the $G_{F}$ anomalies.

(ii) For large $N$, it was proposed [1] that a possible phase can be described by the nonvanishing bi-fermion condensates

$$
\left\langle\psi^{i j} \eta_{j}^{A}\right\rangle=\Lambda^{3}\left(\begin{array}{c}
c \mathbf{1}_{8} \\
\hline \mathbf{0}_{N-8,8}
\end{array}\right)^{i A}, \quad\left\langle\psi^{i k} \chi_{k j}\right\rangle=\Lambda^{3}\left(\begin{array}{ll|ll|l}
a \mathbf{1}_{8} & & & & \\
\hline & d_{1} & & & \\
& & \ddots & & \\
& & & d_{N-12} & \\
\hline & & & b \mathbf{1}_{4}
\end{array}\right)_{j}^{i},
$$

with symmetry breaking

$$
S U(N)_{\mathrm{c}} \times S U(8)_{\mathrm{f}} \times U(1)^{2} \rightarrow S U(8)_{\mathrm{cf}} \times U(1)^{N-11} \times S U(4)_{\mathrm{c}} .
$$

The massless baryons are

$$
\tilde{B}_{j}^{A}=\psi^{i k} \chi_{[k j]} \eta_{i}^{A} \sim \eta_{j}^{A}, \quad(9 \leq j \leq N-4)
$$

and

$$
B^{\{A B\}}=\psi^{i j} \eta_{i}^{A} \eta_{j}^{B}
$$

(iii) Another possible phase, for $N \geq 8$, is described by the condensates,

$$
\left\langle\phi^{i A}\right\rangle=\left\langle\psi^{i j} \eta_{j}^{A}\right\rangle ; \quad\left\langle\tilde{\phi}_{j}^{i}\right\rangle \equiv\left\langle\psi^{i k} \chi_{k j}\right\rangle .
$$


where

$$
\left\langle\psi^{i j} \eta_{j}^{A}\right\rangle=\Lambda^{3}\left(\begin{array}{c}
c \mathbf{1}_{8} \\
\hline \mathbf{0}_{N-8,8}
\end{array}\right)^{i A}, \quad\left\langle\psi^{i k} \chi_{k j}\right\rangle=\Lambda^{3}\left(\begin{array}{c|c|cc}
\mathbf{0}_{8} & & & \\
\hline & d_{1} & & \\
& & \ddots & \\
& & d_{N-8}
\end{array}\right)_{j}^{i}
$$

The symmetry breaking pattern is:

$$
S U(N) \times S U(8) \times U(1)^{2} \rightarrow S U(8)_{c f} \times U(1)^{N-8} .
$$

(iv) Still another option, consistent for any value of $N$, considered in [1], is that the gauge group dynamically Abelianizes completely, by the adjoint condensates

$$
\left\langle\tilde{\phi}_{j}^{i}\right\rangle \equiv\left\langle\psi^{i k} \chi_{k j}\right\rangle=d_{j} \delta_{j}^{i}, \quad \sum_{j} d_{j}=0 ; \quad i, j=1,2, \ldots, N,
$$

with no particular relations among $d_{j}$ 's. We also assume that no color-flavor locking takes place, i.e.,

$$
\left\langle\phi^{i A}\right\rangle=\left\langle\psi^{i j} \eta_{j}^{A}\right\rangle=0 .
$$

The symmetry breaking occurs as:

$$
S U(N)_{\mathrm{c}} \times S U(8)_{\mathrm{f}} \times U(1)^{2} \rightarrow \prod_{\ell=1}^{N-1} U_{\ell}(1) \times S U(8)_{\mathrm{f}} \times \tilde{U}(1)
$$

where $\tilde{U}(1)$ is an unbroken combination of the two nonanomalous $U(1)$ 's, (2.3), with charges:

$$
\psi: 2, \quad \chi:-2, \quad \eta:-1
$$

There are a few more possible dynamical scenarios involving partial color-flavor locking and dynamical Abelianization. $G_{F}$ anomaly matching is satisfied in all cases, except for the first of the above, scenario (ii): it turns out that the anomaly matching involving a discrete symmetry, $Z_{N^{*}} \times S U(8)^{2}$ fails for some $N$. Except for this, there is for the moment no way of deciding which of the dynamical scenarios is actually realized in the infrared.

3. $\left(N_{\psi}, N_{\chi}\right)=(1,0)$ model

$$
\psi^{\{i j\}}, \quad \eta_{i}^{B}, \quad B=1,2, \ldots, N+4,
$$

or

$$
\square+(N+4) \square .
$$

The (continuous) symmetry of this model is

$$
S U(N)_{\mathrm{c}} \times S U(N+4)_{\mathrm{f}} \times U(1) .
$$


where $U(1)$ is an anomaly-free combination of $U_{\psi}(1)$ and $U_{\eta}(1)$, with

$$
Q_{\psi}: \quad N+4 ;, \quad Q_{\eta}: \quad-(N+2) .
$$

There are also discrete symmetries

$$
\mathbb{Z}_{\psi}=\mathbb{Z}_{N+2} \subset U_{\psi}(1), \quad \mathbb{Z}_{\eta}=\mathbb{Z}_{N+4} \subset U_{\eta}(1) .
$$

(i) It was found [4] that 't Hooft anomaly matching conditions alone allow, remarkably, for a confinement phase with no bifermion condensates, i.e., with no chiral symmetry breaking. The candidate massless baryons are:

$$
B^{[A B]}=\psi^{i j} \eta_{i}^{A} \eta_{j}^{B}, \quad A, B=1,2, \ldots, N+4,
$$

\begin{tabular}{|c|c|c|c|}
\hline fields & $S U(N)_{c}$ & $S U(N+4)$ & $\tilde{U}(1)$ \\
\hline$\psi$ & $\square$ & $\frac{N(N+1)}{2} \cdot(\cdot)$ & $N+4$ \\
$\eta^{A}$ & $(N+4) \cdot \square$ & $N \cdot \square$ & $-(N+2)$ \\
\hline$B^{[A B]}$ & $\frac{(N+4)(N+3)}{2} \cdot(\cdot)$ & $\square$ & $-N$ \\
\hline
\end{tabular}

Table 1: Chirally symmetric phase of the $(1,0)$ model

(ii) Another, natural dynamical scenario is a color-flavor locked Higgs phase, in which bifermion condensate

$$
\left\langle\psi^{\{i j\}} \eta_{i}^{B}\right\rangle=C \delta^{j B}, \quad j, B=1,2, \ldots N,
$$

forms, breaking the color dynamically, and in which the symmetry is reduced to

$$
S U(N)_{\mathrm{cf}} \times S U(4)_{\mathrm{f}} \times U^{\prime}(1) .
$$

As this forms a subgroup of the full symmetry group, (3.3), it is quite easily seen, by making the decomposition of the fields in the subgroup, that a subset of the same baryons saturate all of the triangles associated with the reduced symmetry group. See Table 2.

\section{4. $(2,0)$ model}

An interesting generalization of the above is the model with matter fermions

$$
\psi^{\{i j, m\}}, \quad \eta_{i}^{B}, \quad m=1,2, \quad B=1,2, \ldots, 2(N+4),
$$

or

$$
2 \square+2(N+4) \square .
$$

The (continuous) symmetry of this model is

$$
S U(N)_{\mathrm{c}} \times S U(2)_{f} \times S U(2 N+8)_{\mathrm{f}} \times U(1),
$$




\begin{tabular}{|c|c|c|c|}
\hline fields & $S U(N)_{c f}$ & $S U(4)_{f}$ & $U^{\prime}(1)$ \\
\hline$\psi$ & $\square$ & $\frac{N(N+1)}{2} \cdot(\cdot)$ & 1 \\
$\eta^{A_{1}}$ & $\square$ & & \\
$\eta^{A_{2}}$ & $4 \cdot \square$ & $N \cdot \square$ & $-\frac{1}{2}$ \\
\hline$B^{\left[A_{1} B_{1}\right]}$ & $\square$ & $N^{2} \cdot(\cdot)$ & -1 \\
$B^{\left[A_{1} B_{2}\right]}$ & $4 \cdot \square$ & $N \cdot \square$ & $-\frac{N}{2}$ \\
\hline
\end{tabular}

Table 2: Color-flavor locked phase in the $(1,0)$ model. $A_{1}$ or $B_{1}$ stand for $A, B=1,2, \ldots, N . A_{2}$ or $B_{2}$ the rest of the flavor indices.

where $U(1)$ is an anomaly-free combination of $U_{\psi}(1)$ and $U_{\eta}(1)$,

$$
U(1): \quad \psi \rightarrow e^{i \alpha / 2(N+2)} \psi, \quad \eta \rightarrow e^{-i \alpha / 2(N+4)} \eta .
$$

It turns out that, in contrast to the $(1,0)$ model, it is not possible to find a confining, chirally symmetric phase with no fermion condensates: there are no candidate massless, color-singlet baryons made out of $\psi^{\{i j, m\}}$ and $\eta_{i}^{B}$ which could saturate the anomalies.

Instead, we find that the system could flow into a double $S U(N)$ color-flavor-flavor locked phase, with condensates,

$$
\begin{gathered}
\left\langle\psi^{\{i j, 1\}} \eta_{j}^{B}\right\rangle=C \delta^{i, B}, \quad j, B=1,2, \ldots N, \\
\left\langle\psi^{\{i j, 2\}} \eta_{j}^{B}\right\rangle=C \delta^{i, B-N}, \quad j=1,2, \ldots N, \quad B=N+1, \ldots, 2 N
\end{gathered}
$$

The symmetry is

$$
S U(N)_{c} \times S U(2)_{f} \times S U(2 N+8)_{f} \times U(1)_{f} \rightarrow S U(N)_{c f} \times \tilde{U}(1) \times S U(2)_{f f} \times S U(8),
$$

where $S U(2)_{f f}$ is a linear combination of $S U(2)_{f}$ and

$$
S U(2) \subset S U(2 N) \subset S U(2 N+8)
$$

which exhange the first and second $N$ flavors. The charges of the unbroken $S U(2)$ are:

$$
\left(\begin{array}{c}
\psi^{i j, 1} \\
\psi^{i j, 2}
\end{array}\right) \sim \underline{2} ; \quad\left(\begin{array}{c}
\eta_{i}^{A \leq N} \\
\eta_{i}^{N \leq A \leq 2 N}
\end{array}\right) \sim \underline{2}^{*}
$$

the $\tilde{U}(1)$ charges are as before,

$$
\psi: \quad 1 ; \quad \eta^{B \leq 2 N}: \quad-1 ; \quad \eta^{B>2 N}: \quad-\frac{1}{2} .
$$

The baryons are

$$
B^{A, C}=\psi^{i j, 1} \eta_{i}^{A \leq N} \eta_{j}^{C}+\psi^{i j, 2} \eta_{i}^{N<A \leq 2 N} \eta_{j}^{C} ; \quad C>2 N
$$


which is a $S U(2)$ singlet; the others are

$$
B^{\left[A_{1} B_{1}\right], 1}=\psi^{i j, 1} \eta_{i}^{A_{1}} \eta_{j}^{B_{1}}, \quad A_{1}, B_{1}=1,2, \ldots, N
$$

and

$$
B^{\left[A_{2} B_{2}\right], 2}=\psi^{i j, 2} \eta_{i}^{A_{2}} \eta_{j}^{B_{2}}, \quad A_{2}, B_{2}=N+1, N+2, \ldots, 2 N
$$

which form a doublet. Their $\tilde{U}(1)$ charges are:

$$
B^{A, C}: \quad-\frac{1}{2} ; \quad B^{[A B], m}:-1 .
$$

\begin{tabular}{|c|c|c|c|c|}
\hline fields & $S U(N)_{c f}$ & $S U(8)$ & $S U(2)$ & $\tilde{U}(1)$ \\
\hline$\psi$ & $2 \cdot \square$ & $N(N+1) \cdot(\cdot)$ & $\frac{N(N+1)}{2} \cdot \square$ & 1 \\
$\eta^{A_{i}}$ & $2 \cdot(\square \oplus \square)$ & $2 N^{2} \cdot(\cdot)$ & $N^{2} \cdot \square$ & -1 \\
$\eta^{C}$ & $8 \cdot \square$ & $N \cdot \square$ & $8 N \cdot(\cdot)$ & $-\frac{1}{2}$ \\
\hline$B^{A, C}$ & $8 \cdot \square$ & $N \cdot \square$ & $8 N \cdot(\cdot)$ & $-\frac{1}{2}$ \\
$B^{\left[A_{i} B_{i}\right], m}$ & $2 \cdot \square$ & $N(N-1) \cdot(\cdot)$ & $\frac{N(N-1)}{2} \square$ & -1 \\
\hline
\end{tabular}

Table 3: An $S U$ (2) flavor-flavor locked symmetric phase in the $(2,0)$ model. $A_{i}$ or $B_{i}(i=1,2)$ indicate the flavor indices up to $2 N ; C$ the rest, $2 N+1, \ldots, 2 N+8$.

\section{5. $(0,1)$ model}

This model was also studied by by Appelquist-Duan-Sannino [4], by Poppitz [6] and by ourselves [1] earlier. The matter fermions are

$$
\chi_{[i j]}, \quad \tilde{\eta}^{B j}, \quad B=1,2, \ldots,(N-4) .
$$

The symmetry is

$$
S U(N)_{\mathrm{c}} \times S U(N-4)_{\mathrm{f}} \times U(1)
$$

where the anomaly free $U(1)$ charge is

$$
\begin{gathered}
\chi: N-4 ; \quad \tilde{\eta}^{B j}: \quad-(N-2) . \\
b_{0}=11 N-(N-2)-(N-4)=9 N+6 .
\end{gathered}
$$

There are also discrete symmetries

$$
\mathbb{Z}_{\chi}=\mathbb{Z}_{N-2} \subset U_{\psi}(1), \quad \mathbb{Z}_{\eta}=\mathbb{Z}_{N-4} \subset U_{\eta}(1) .
$$




\begin{tabular}{|c|c|c|c|}
\hline fields & $S U(N)_{c}$ & \multicolumn{1}{l|}{$S U(N-4)$} & $U(1)$ \\
\hline & $\square$ & \multicolumn{2}{|c|}{} \\
$\chi$ & $\square$ & $\frac{N(N-1)}{2} \cdot(\cdot)$ & $N-4$ \\
$\tilde{\eta}^{A}$ & $(N-4) \cdot \square$ & $N \cdot \square$ & $-(N-2)$ \\
\hline$B^{\{A B\}}$ & $\frac{(N-4)(N-3)}{2} \cdot(\cdot)$ & $\square$ & $-N$ \\
\hline
\end{tabular}

Table 4: Confinement and unbroken symmetry in the $(0,1)$ model

(i) As in the $(1,0)$ model discussed earlier, the anomaly matching argument alone allows a chirally symmetric (no fermion condensates) confining vacuum, with massless baryons

$$
B^{\{C D\}}=\chi_{[i j]} \tilde{\eta}^{i C} \tilde{\eta}^{j D}, \quad C, D=1,2, \ldots(N-4),
$$

assumed to be symmetric in $C D$. See Table 4 .

(ii) Another natural hypothesis is that this system develops a condensate of the form

$$
\left\langle\chi_{[i j]} \tilde{n}^{B j}\right\rangle=\text { const. } \Lambda^{3} \delta_{i}^{B} ; \quad i, B=1,2, \ldots, N-4,
$$

namely,

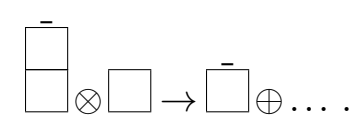

The symmetry is broken as

$$
S U(N)_{\mathrm{c}} \times S U(N-4)_{\mathrm{f}} \times U(1) \rightarrow S U(N-4)_{\mathrm{cf}} \times U(1)^{\prime} \times S U(4)_{\mathrm{c}} .
$$

A subset of the massless baryons (5.6) saturate all the anomalies associated with $S U(N-$ $4)_{\mathrm{cf}} \times U(1)^{\prime}$. See Table 5. As noted by Appelquist, Duan, Sannino [4], there remains the $\chi_{i_{2} j_{2}}$ fermions which remain massless and strongly coupled to the $S U(4)_{c}$. We may assume that $S U(4)_{c}$ confines, and the condensate

$$
\langle\chi \chi\rangle \neq 0
$$

in

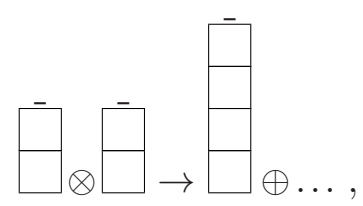

forms and $\chi_{i_{2} j_{2}}$ acquire dynamically mass.

\section{Conclusion}

Many other systems have been analyzed $((1,1),(1,0),(2,0),(3,0)(0,1),(0,2)(0,3),(2,1)$, $(1,-1)$, etc. $)$ and in more details. Let us summarize some of the lessons learned: 


\begin{tabular}{|c|c|c|c|}
\hline fields & $S U(N-4)_{c f}$ & $U^{\prime}(1)$ & $S U(4)_{c}$ \\
\hline & $\square$ & & $(N-4)(N-5)$ \\
$\chi_{i_{1} j_{1}}$ & $\square$ & $N$ & $\frac{(\cdot)}{2}$ \\
$\chi_{i_{1} j_{2}}$ & $4 \cdot \square$ & $\frac{N}{2}$ & $(N-4) \cdot \square$ \\
& & & $\square$ \\
$\chi_{i_{2} j_{2}}$ & $\frac{4 \cdot 3}{2} \cdot(\cdot)$ & 0 & $\square$ \\
$\tilde{\eta}^{A, i_{1}}$ & $\square$ \\
$\tilde{\eta}^{A, i_{2}}$ & $4 \cdot \square$ & $-N$ & $(N-4)^{2} \cdot(\cdot)$ \\
\hline$B^{\{A B\}}$ & $\square$ & $-\frac{N}{2}$ & $(N-4) \cdot \square$ \\
\hline
\end{tabular}

Table 5: Color-flavor locking in the $(0,1)$ model. The color index $i_{1}$ or $j_{1}$ runs up to $N-4$. The rest is indicated by $i_{2}$ or $j_{2}$.

1. Confining vacuum without chiral symmetry breaking turns out to be rather exceptional (only $(1,0)$ and $(0,1)$ models allow the matching with such a hypothesis); the dynamical Higgs phase seems to appear equally in many models, and perhaps, more natural.

2. In many models color-flavor (or even color-flavor-flavor) locking hypothesis allows one to achieve the anomaly matching.

3. Dynamical Abelianization $S U(N) \rightarrow U(1)^{N-1}$ (full or partial) due to some bifermion condensate in adjoint representation, is another important mechanism for many systems to be described consistently in the infrared.

4. Complementarity [10] may or may not work.

5. The large $N$ planar equivalence to supersymmetric $S U(N)$ Yang-Mills does not hold in the $\psi \chi \eta$ model (cfr. [9]).

6. $a$-theorem is always satisfied in the proposed dynamical scenarios, whereas the ACS criterion [4] sometimes fails.

\section{References}

[1] S. Bolognesi, K. Konishi and M. Shifman, Patterns of symmetry breaking in chiral QCD, Phys. Rev. D 97, no. 9, 094007 (2018) doi:10.1103/PhysRevD.97.094007 [arXiv:1712.04814 [hep-th]].

[2] G. 't Hooft, Naturalness, Chiral Symmetry, and Spontaneous Chiral Symmetry Breaking, in Recent Developments In Gauge Theories, Eds. G. 't Hooft, C. Itzykson, A. Jaffe, H. Lehmann, P. K. Mitter, I. M. Singer and R. Stora, (Plenum Press, New York, 1980) [Reprinted in Dynamical Symmetry Breaking, Ed. E. Farhi et al. (World Scientific, Singapore, 1982) p. 345 and in G. 't Hooft, Under the Spell of the Gauge Principle, (World Scientific, Singapore, 1994), p. 352].

[3] Z. Komargodski and A. Schwimmer, On Renormalization Group Flows in Four Dimensions, JHEP 1112, 099 (2011) doi:10.1007/JHEP12(2011)099 [arXiv:1107.3987 [hep-th]]. 
[4] T. Appelquist, A. G. Cohen, M. Schmaltz and R. Shrock, New constraints on chiral gauge theories, Phys. Lett. B 459, 235 (1999) [hep-th/9904172]; T. Appelquist, Z. y. Duan and F. Sannino, Phases of chiral gauge theories, Phys. Rev. D 61, 125009 (2000) [hep-ph/0001043]; Y. L. Shi and R. Shrock, $A_{k} \bar{F}$ chiral gauge theories, Phys. Rev. D 92,105032 (2015) [arXiv:1510.07663 [hep-th]]; Y. L. Shi and R. Shrock, Renormalization-Group Evolution and Nonperturbative Behavior of Chiral Gauge Theories with Fermions in Higher-Dimensional Representations, Phys. Rev. D 92, 125009 (2015) [arXiv:1509.08501 [hep-th]].

[5] Stuart Raby, Savas Dimopoulos, and Leonard Susskind, Tumbling Gauge Theories, Nucl. Phys. B169, 373 (1980).

[6] E. Poppitz and Y. Shang, Chiral Lattice Gauge Theories Via Mirror-Fermion Decoupling: A Mission (im)Possible?, Int. J. Mod. Phys. A 25, 2761 (2010) [arXiv:1003.5896 [hep-lat]].

[7] E. Eichten, R. D. Peccei, J. Preskill and D. Zeppenfeld, Chiral Gauge Theories in the 1/n Expansion, Nucl. Phys. B 268, 161 (1986).

[8] J. Goity, R. D. Peccei and D. Zeppenfeld, Tumbling and Complementarity in a Chiral Gauge Theory,' Nucl. Phys. B 262, 95 (1985). doi:10.1016/0550-3213(85)90065-3.

[9] A. Armoni and M. Shifman, A Chiral SU(N) Gauge Theory Planar Equivalent to Super-Yang-Mills, Phys. Rev. D 85, 105003 (2012) [arXiv:1202.1657 [hep-th]].

[10] E. H. Fradkin and S. H. Shenker, Phase Diagrams of Lattice Gauge Theories with Higgs Fields, Phys. Rev. D 19 (1979) 3682. 